\title{
THE FEKETE-SZEGÖ INEQUALITY \\ BY A VARIATIONAL METHOD
}

\author{
ALBERT PFLUGER
}

1. Denote by $S$, as customary, the class of normalized univalent functions

$$
f(z)=z+a_{2} z^{2}+a_{3} z^{3}+\ldots
$$

from the unit disk $\boldsymbol{D}$ into $\boldsymbol{C}$. Denote by $S_{2}$ the class of odd functions $f_{2}$ in $S$. When Littlewood and Paley [3] proved that the coefficients in

$$
f_{2}(z)=z+b_{3} z^{3}+b_{5} z^{5}+\ldots, f_{2} \in S_{2},
$$

have a universal bound $B$ and presumed $B$ to be 1, Fekete and Szegö [1] were quick to disprove this conjecture. Observing that the square root operation

$$
f_{2}(z)=\sqrt{f\left(z^{2}\right)}=z+\frac{a_{2}}{2} z^{3}+\frac{1}{2}\left(a_{3}-\frac{1}{4} a_{2}^{2}\right) z^{5}+\ldots
$$

effects a mapping of $S$ onto $S_{2}$ and using Loewner's differential equation, they found for $\left|a_{3}-(1 / 4) a_{2}^{2}\right|$ the exact bound over $S$, hence $1 / 2+e^{-2 / 3}=1,013 \ldots$ for the maximum of $\left|b_{5}\right|$ over $S_{2}$. This method works for all functionals $\left|a_{3}-\lambda a_{2}^{2}\right|$, where $0 \leqq \lambda<1$, and leads to the sharp inequality

$$
\left|a_{3}-\lambda a_{2}^{2}\right| \leqq 1+2 \exp \frac{2 \lambda}{\lambda-1}, \quad f \in S,
$$

where $0 \leqq \lambda<1$. This is known as the Fekete-Szegö theorem.

However, besides $a_{2}$ and $a_{3}$ being real, the method does not give any information about functions yielding equality in (1). It is the purpose of this paper to give a full treatment of the Fekete-Szegö theorem by variational method and thus get a precise description of the image domain under extremal functions.

2. We denote by $\Phi$ the functional which is defined by $\Phi(f)=a_{3}-\lambda a_{2}^{2}, \lambda \in \boldsymbol{C}$. Its range over $S$ is a closed disk centered at 0 , and to determine its radius it suffices to find $\max _{S} \operatorname{Re} \Phi$. For functions $f$ yielding this maximum $\Phi(f)$ is obviously positive. The main problem of this paper is to maximize $\operatorname{Re} \Phi$ over $S$, where $0<\lambda<1$.

We assume $f$ to be extremal and apply a standard variational technique. Since the Fréchet differential of $\Phi$ at $f$ is given by

$$
L(g)=b_{3}-2 a_{2} \lambda \cdot b_{2}, g(z)=z+b_{2} z^{2}+b_{3} z^{3}+\ldots,
$$


and

it follows

$$
L\left(\frac{f^{2}}{f-w}\right)=-\frac{1+2 a_{2}(1-\lambda) w}{w^{2}}
$$

$$
-\frac{1+2 a_{2}(1-\lambda) w}{w^{2}}\left(\frac{d w}{w}\right)^{2}>0 \quad \text { along } \quad \partial f(D)
$$

as a necessary condition for $f$ to be extremal. More precisely, $f(\boldsymbol{D})$ is dense in $\boldsymbol{C}$ and its boundary consists of finitely many analytic arcs such that (2) holds along their interiors.

In this condition the coefficient $a_{2}$ of the extremal function $f$ is an unknown parameter. But using the method of Fekete and Szegö one easily gets $a_{2}=$ $\pm(2 /(1-\lambda)) e^{-\lambda /(1-\lambda)}$, and this opens the road to a full discussion of the extremal domain $f(\boldsymbol{D})$. Actually, starting from (2) with $\lambda=1 / 4$ Schaeffer and Spencer [5] set up Schiffer's differential equation of $f$ and proved that the extremal function is unique, up to a rotation through $180^{\circ}$, and maps $\boldsymbol{D}$ onto a domain which is bounded by a forked slit consisting of the radial half line from $\infty$ to $(1 / 4) e^{-1 / 3}$ and two arcs issuing from $(1 / 4) e^{-1 / 3}$ which are symmetric to the real axis, or what one gets by a rotation through $180^{\circ}$.

Though this procedure works for all $\lambda, 0<\lambda<1$, we will follow below a method Garabedian and Schiffer [2] used for the coefficient $a_{3}$. It allows a direct access to condition (2) and gives a clear insight into what is going on geometrically.

3. We first show that $a_{2} \neq 0$ for each extremal $f$. In fact, for $a_{2}=0$ condition (2) becomes $\left(d w / w^{2}\right)^{2}<0$ and implies that the omitted set of $f$ must lie on the imaginary axis and hence $f$ be of the form

$$
f(z)=\frac{z}{1+2 \mu i z-z^{2}}, \quad-1 \leqq \mu \leqq 1 .
$$

For all these functions (one of which has $a_{2}=0$ ) we have $\Phi(f)=1$. But (1) implies $\max _{S} \operatorname{Re} \Phi>1$ for $0<\lambda<1$ showing that $a_{2} \neq 0$ for each $f$ maximizing $\operatorname{Re} \Phi$ over $S$.

This last step apparently makes use of the Fekete-Szegö theorem. In Section 5, however, the extremal function $f$ appears as a solution of (11), and this equation may be set up directly without appealing to the extremal property of its solution. Nevertheless, an elementary example with $\Phi(f)>1$ for $0<\lambda<1$ would be welcome.

By the same argument as above the functions (3) are extremal for $\operatorname{Re} \Phi$ if $\lambda=1$. One can show that for each $\lambda \in C \backslash\{1\}$ a function $f$ maximizing $\operatorname{Re} \Phi$ has its coefficient $a_{2}$ non-vanishing.

Since $a_{2}(1-\lambda) \neq 0$, we set

$$
\alpha_{1}=2 a_{2}(\lambda-1)=i r e^{i \alpha}, \quad r>0, \quad \alpha \in R .
$$

It will prove convenient to introduce a new function

$$
\varphi(z)=\alpha_{1} z+\alpha_{2} z^{2}+\alpha_{3} z^{3}+\ldots
$$


and a new variable $\omega$ by setting

$$
\varphi=\alpha_{1} f \text { and } \omega=\alpha_{1} w .
$$

Thus we obtain

$$
\frac{1-2 a_{2}(\lambda-1) w}{w^{2}}\left(\frac{d w}{w}\right)^{2}=-r^{2} e^{-2 i \alpha} \cdot \frac{1-\omega}{\omega^{2}}\left(\frac{d \omega}{\omega}\right)^{2}
$$

and

$$
-e^{-2 i \alpha} \frac{1-\omega}{\omega^{2}}\left(\frac{d \omega}{\omega}\right)^{2} \leqq 0
$$

along $\partial \varphi(\boldsymbol{D})$ as the necessary condition for the domain $\varphi(\boldsymbol{D})$.

4. The quadratic differential

$$
\frac{1-\omega}{\omega^{2}}\left(\frac{d \omega}{\omega}\right)^{2}
$$

appearing in (7) will play an important role. We call its $\alpha$-trajectory the trace of a maximal solution to the differential equation

$$
\frac{1-\omega}{\omega^{2}}\left(\frac{d \omega}{\omega}\right)^{2}=e^{2 i \alpha} \cdot d t^{2}
$$

where $t$ is a real parameter, and will now determine the $\alpha$-trajectories that emanate from $\infty$.

To find them we fix in the upper half plane the branch of $(1-\omega)^{1 / 2}$ which is 1 at 0 . The Schwarz-Christoffel integral

$$
J(\omega)=\int_{\infty}^{\omega} \frac{(1-\omega)^{1 / 2}}{\omega^{2}} d \omega
$$

is univalent in the upper half plane and takes it onto that part of $\boldsymbol{C}$ which is above the broken line shown in the nearby diagram.
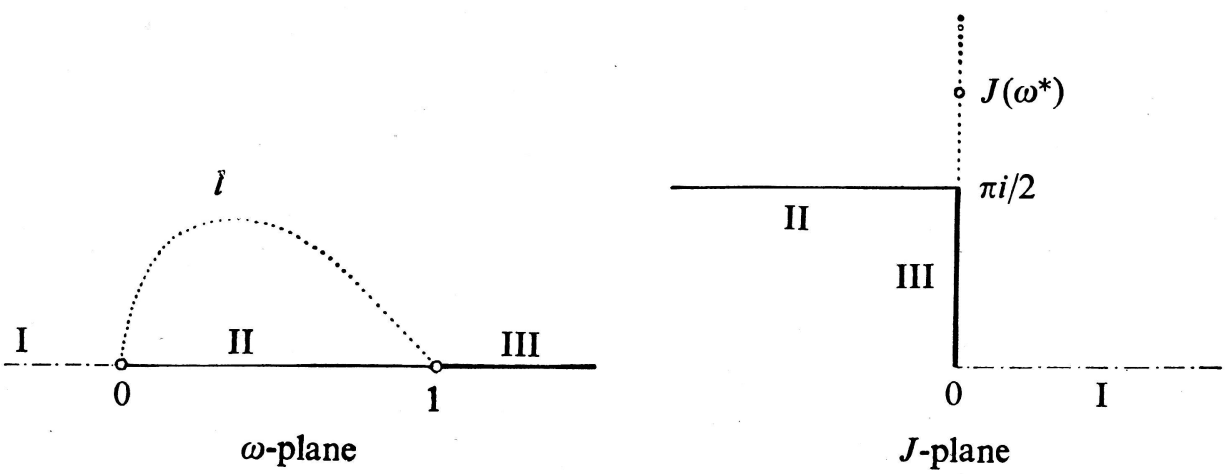

Roman marks correspond to sides. 
By reflection in the half line I we extend $J$ to a univalent mapping from the $\omega$-plane cut along the positive real axis onto $C$ minus the horizontal half strip with the corners $J= \pm \pi i / 2$; it takes $\infty$ onto 0 and the two boundary elements at 1 onto $\pi i / 2$ and $-\pi i / 2$, respectively. The inverse mapping $J^{-1}$ takes the open half line $J=e^{i \alpha} \cdot t, t>0$, for $|\alpha|<\pi / 2$ onto a simple analytic arc that emanates from $\infty$ and terminates at 0 . It will be denoted by $\Gamma_{\alpha}$. For $\alpha=0$ it is the half line $I$ in the $\omega$-plane; for $0<\alpha<\pi / 2 \quad(0>\alpha>-\pi / 2)$ it passes through the upper (lower) half plane. It has the asymptotic half line

$$
\omega=-e^{-2 i \alpha} \cdot \tau-1 / 3, \quad \tau>0,
$$

and terminates at 0 with the tangent vector $e^{-i \alpha}$.

The mapping $J^{-1}$ takes the dotted line up from $\pi i / 2$ along the imaginary axis onto an arc $l$ that issues from $\omega=1$ under the angle $2 \pi / 3$ with the positive real axis and terminates at 0 in vertical direction. Denote its complex conjugate by $l$. Then the closure of the arcs $l$ and $\bar{l}$, together with the half line III, is the boundary of a domain $\Omega$ which is the one to one image under $J^{-1}$ of the right half plane. Through each point of $\Omega$ passes one and only one arc $\Gamma_{\alpha},|\alpha|<\pi / 2$; the boundary of $\Omega$ is denoted by $\Gamma^{*}$.

Now we make use of the trick of Garabedian and Schiffer [2]. By condition (7) the boundary of the domain $\varphi(D)$ is an unbounded continuum on some $\Gamma_{\alpha}$ $-\pi / 2<\alpha<\pi / 2$ or on $\Gamma^{*}$. The integral $\int_{0}^{2 \pi} \frac{1}{\varphi\left(e^{i \theta}\right)} \cdot \frac{d \theta}{2 \pi}$ is the conformal center of gravity (G. Pólya and G. Szegö [4]) of the set omitted by $1 / \varphi$ and therefore is contained in its convex hull. The residue theorem and (5) yield

$$
\frac{1}{2 \pi} \int_{0}^{2 \pi} \frac{d \theta}{\varphi\left(e^{i \theta}\right)}=\frac{1}{2 \pi i} \int_{\partial D} \frac{d z}{z \varphi(z)}=-\frac{\alpha_{2}}{\alpha_{1}^{2}}=\frac{1}{2(1-\lambda)},
$$

indicating that the omitted set of $1 / \varphi$ has its conformal center on the positive real axis and so excluding $|\alpha|<\pi / 2$, i.e., according to (4) $\alpha_{1}$ must be real (and $\neq 0$ ). By a rotation we may always assume that $\alpha_{1}>0$ and reserve $-\alpha_{1}$ to a second solution. By (4) it follows $a_{2}<0$ and a comparison of coefficients in (6) implies $2 \alpha_{2}(\lambda-1)=\alpha_{1}^{2}$, hence $\alpha_{2}<0$. Since $a_{3}-\lambda a_{2}^{2}$ is positive in the extremal case, it follows $a_{3}>0$ and then, by $\alpha_{3} / \alpha_{1}=a_{3}$, that $\alpha_{3}>0$. We conclude that all three coefficients $\alpha_{1}, \alpha_{2}, \alpha_{3}$ are real if $f=\varphi / \alpha_{1}$ is extremal.

5. To determine the coefficients $\alpha_{1}, \alpha_{2}$ and $\alpha_{3}$ explicitly we now use the fact that $\varphi$ is a mapping from the unit disk. Transplantation of $\left((1-\omega) / \omega^{2}\right)(d \omega / \omega)^{2}$ with $\varphi$ into the unit disk yields

$$
R(z)\left(\frac{d z}{z}\right)^{2}=\frac{1-\varphi(z)}{\varphi^{2}(z)}\left(\frac{d \varphi(z)}{\varphi(z)}\right)^{2}
$$

i.e., Schiffer's differential equation of $\varphi$. By the preceding section the boundary of $\varphi(\boldsymbol{D})$ lies on $\Gamma^{*}$ and has at 0 a mapping radius $\alpha_{1}$. From (4) and $0<\lambda<1$ it follows 
that $0<\alpha_{1}<4$, and the omitted continuum therefore contains (besides the half line $[1, \infty))$ some portions of the arcs $l$ and $l$, one of which might be empty. The function $R(z)$ has at 0 a double pole and two double zeros at the points $e^{i \beta}$ and $e^{i \gamma}$ which correspond to the two tips on $l$ and $\bar{l}$ or to the one tip and to $\omega=1$, respectively. The condition (7) implies $R(z) \geqq 0$ on the unit circle $T$ and by the Schwarz reflection principle we infer

$$
R(z)=k\left(\frac{\left(z-e^{i \beta}\right)\left(z-e^{i \gamma}\right)}{z}\right)^{2}
$$

From $R(z) \geqq 0$ on $T$ it follows $k e^{i(\beta+\gamma)}>0$. Comparing the two sides of (9) at $z=0$ we obtain $\alpha_{1}^{2} k e^{2 i(\beta+\gamma)}=1$; hence $\gamma=-\beta$ and $k=1 / \alpha_{1}^{2}$.

Taking square roots and setting $\omega=\varphi(z)$ we obtain from this

$$
\alpha_{1} \frac{(1-\omega)^{1 / 2}}{\omega^{2}} d \omega=\frac{\left(z-e^{i \beta}\right)\left(z-e^{-i \beta}\right)}{z^{2}} d z
$$

and by integration

$$
\alpha_{1} J_{1}(\omega)=F(z)
$$

where by (8)

$$
J_{1}(\omega)=J(\omega)-J(1)=-\frac{(1-\omega)^{1 / 2}}{\omega}+\frac{1}{2} \log \frac{1+(1-\omega)^{1 / 2}}{1-(1-\omega)^{1 / 2}}
$$

and

$$
F(z)=z-\frac{1}{z}-2 \cos \beta \cdot \log z+c
$$

with $c$ a suitable constant. Using the expansion

$$
J_{1}(\omega)=-\frac{1}{\omega}+\frac{1}{2}+\log 2-\frac{1}{2} \log \omega-\frac{\omega}{8}+O\left(\omega^{2}\right)
$$

for $\omega \rightarrow 0$, inserting (5) for $\omega$ and eliminating $\alpha_{2}$ by $\alpha_{1}^{2}=2 \alpha_{2}(\lambda-1)$, we obtain at 0 the expansion

$$
\alpha_{1} J_{1}(\varphi(z))=-\frac{1}{z}+A^{\prime} \log z+A_{0}+A_{1} z+O\left(z^{2}\right)
$$

where

$$
\left\{\begin{array}{l}
A^{\prime}=-\alpha_{1} / 2 \\
A_{0}=\frac{\alpha_{1}}{2}\left(\frac{\lambda}{\lambda-1}-\log \alpha_{1} / 4\right) \\
A_{1}=\frac{\alpha_{3}}{\alpha_{1}}-\frac{\alpha_{1}^{2}}{8} \frac{1+\lambda^{2}}{(\lambda-1)^{2}}
\end{array}\right.
$$


Comparison of the coefficients in (11) yields

$$
\begin{aligned}
& \alpha_{1}=4 \cos \beta, \\
& \frac{\alpha_{1}}{2}\left(\frac{\lambda}{\lambda-1}-\log \alpha_{1} / 4\right)=c, \\
& \frac{\alpha_{3}}{\alpha_{1}}-\frac{\alpha_{1}^{2}}{8} \frac{1+\lambda^{2}}{(1-\lambda)^{2}}=1 .
\end{aligned}
$$

There are three or two points on $T$ which are taken onto $\omega=1$ under $\omega=\varphi(z)$. If $z=e^{i \delta}$ is one of them, then by (12) and (13) the equation $\alpha_{1} J_{1}(1)=F\left(e^{i \delta}\right)$ becornes

$$
0=2 i \sin \delta-2 i \delta \cos \beta+c
$$

and shows that $c$ must be imaginary. The second equation of (15) then indicates that $c$ is real, hence zero, and this equation reduces further to

$$
\alpha_{1}=4 \exp \frac{\lambda}{\lambda-1}
$$

With $a_{3}=\alpha_{3} / \alpha_{1}$ and (4) the third equation of (15) gives

hence

$$
a_{3}-\lambda a_{2}^{2}=\frac{\alpha_{3}}{\alpha_{1}}-\frac{\lambda}{4(1-\lambda)^{2}} \alpha_{1}^{2}=1+\alpha_{1}^{2} / 8
$$

$$
a_{3}-\lambda a_{2}^{2}=1+2 \exp \frac{2 \lambda}{\lambda-1},
$$

which is the maximum of $\operatorname{Re} \Phi$ over $S$.

6. By the preceding relations it is easy to see that the solution $\varphi$ (with $\alpha_{1}>0$ ) is unique and all its coefficients are real. In fact, $\lambda$ determines $\alpha_{1}$ by (16) and the equation (11) has a unique solution for $\omega=\varphi(z)$. Moreover, since $J_{1}$ and $F$ are real for $0<\omega<1$ and $z>0, \varphi(z)$ has all coefficients real and the domain $\varphi(\boldsymbol{D})$ is symmetric with respect to the real axis.

As already mentioned the boundary of $\varphi(D)$ is made up of the half line $\omega=1+t$, $t \geqq 0$, and of two portions on the arcs $l$ and $\bar{l}$ which we know now to be symmetric. Let the finite tip with positive imaginary part be denoted by $\omega^{*}$. It is given by $\omega^{*}=$ $\varphi\left(e^{i \beta}\right)$ since $d \omega / d z$ vanishes at $e^{ \pm i \beta}$. By (11) we have

$$
\alpha_{1} J_{1}\left(\omega^{*}\right)=F\left(e^{i \beta}\right)=2 i(\sin \beta-\beta \cos \beta),
$$

and by (15) and (12),

$$
J\left(\omega^{*}\right)=\frac{\pi i}{2}+\frac{1}{2}(\operatorname{tg} \beta-\beta) i,
$$

where $\cos \beta=\alpha_{1} / 4=\exp (\lambda /(\lambda-1))$. This determines $J\left(\omega^{*}\right)$ explicitly and we conclude that the boundary of $\varphi(D)$ is in the preimage under $J$ of the straight segment 
from $J\left(\omega^{*}\right)$ to $\overline{J\left(\omega^{*}\right)}$. (See diagram.) Thus, the domain $\varphi(\boldsymbol{D})$ and the function $\varphi$ are fully described. The relations (6) finally give the extremal function $f$ in $S$ which for $a_{2}<0$ is unique, and the corresponding extremal domain, i.e., $f(z)=\left(1 / \alpha_{1}\right) \varphi(z)$, and $f(\boldsymbol{D})$ is obtained from $\varphi(\boldsymbol{D})$ by the similarity transformation $\omega \mapsto w=\omega / \alpha_{1}$. Note that $0<\alpha_{1} \leqq 4$.

There is a second solution to (10), say $\varphi_{-}$, the unique one which corresponds to $\alpha_{1}=-\left|\alpha_{1}\right|$ and is given by $\varphi_{-}(z)=\varphi(-z)$. Hence $f_{-}(z)=-f(-z)$ is the second function in $S$ that maximizes $\operatorname{Re}\left\{a_{3}-\lambda a_{2}^{2}\right\}$.

For $\lambda=0$ we have the problem of maximizing $\operatorname{Re} a_{3}$ over $S$. In this case $\partial \varphi(\boldsymbol{D})$ is made up only by the radial half line from 1 to $\infty$, and $\partial f(\boldsymbol{D})$ by the half line from $1 / 4$ to $\infty$ as one knows, of course. For $\lambda<0$ we have $\alpha_{1}=4(1+|\lambda|)$. So $\partial \varphi(D)$ is the half-line $\omega=(1+|\lambda|)+t, t \geqq 0$, and renormalizing we again obtain for $f$ a Koebe function. For $\lambda>1$, however, according to Section 4, the conformal center of gravity of the set omitted by $1 / \varphi$ is on the negative real axis; hence $\alpha=0$, $\alpha_{1}$ imaginary, $\partial \varphi(D)$ on $\Gamma_{0}$ from $1-\lambda$ to $\infty$ and $f$ the Koebe function $-i k(i z)$ or $i k(-i z)$.

Remark that it is the same quadratic differential which in all these problems plays the key role in finding the extremal domains.

So far the extremal problem is completely solved for $\lambda$ real. For a non-real $\lambda$ one might start in the same way. Since $a_{2}$ is again non-zero for an extremal function one gets the same condition (7) for the extremal domain $\varphi(D)$. But the difficulty with the unknown parameter $a_{2}$ is now quite serious. We have only a very partial result showing that not all extremal domains have their boundary forked. Note that we call $\partial \varphi(D)$ forked if it contains the radial half line from $\infty$ to 1 and portions on $l$ and $l$ which need not to be symmetric and one of which might be empty. Let $f$ maximize $\operatorname{Re} \varphi$ for some $\lambda$ non-real. Define $\varphi$ and $\omega$ as in (6). We will give a necessary condition for $\lambda$ such that $\partial \varphi(D)$ is forked at $\omega=1$, i.e., $\alpha= \pm \pi i / 2$ and $0<\alpha_{1}<4$. In the same way as in Section 5 it follows that $c$ in (15) must be imaginary, say $c=2 i \tau$, and one easily gets bounds for $\tau$. If $z=e^{i \theta}$ moves along the unit circle, then $\varphi\left(e^{i \theta}\right)$ passes three times through $\omega=1$, or twice (if $\partial \varphi(\boldsymbol{D})$ has only one finite tip), i.e., according to (11), the equation

$$
F\left(e^{i \theta}\right)=2 i(\sin \theta-\theta \cdot \cos \beta+\tau)=0, \quad 4 \cos \beta=\alpha_{1},
$$

must have three (or two) solutions for $\theta$. But the function $h(\theta)=\sin \theta-\theta \cos \beta$ reaches on the interval $[-\pi, \pi]$ its maximum at $\theta=-\beta$ and its minimum at $\theta=\beta$. This gives for $\tau$ the condition $|\tau| \leqq \sin \beta-\beta \cos \beta$. From (15) we infer that

$$
\frac{\lambda}{\lambda-1}=\log \frac{\alpha_{1}}{4}+\frac{4 i \tau}{\alpha_{1}}
$$

hence setting $4 \tau / \alpha_{1}=\sigma$ we obtain

$$
\frac{\lambda}{\lambda-1}=\log \cos \beta+i \sigma, \quad|\sigma| \leqq \operatorname{tg} \beta-\beta
$$


as a necessary condition for $\lambda$ such that the corresponding domain $\varphi(D)$ has its boundary forked. Let $F$ denote the set of the $\lambda$ which satisfy (18). Then for $\lambda \in C \backslash F$ the boundary of the corresponding extremal domain consists of a single analytic slit.

On the other hand, if for some $\lambda$ the extremal domain has a forked boundary, then $\lambda$ is in $F$ and (17) implies $\alpha_{1}=4|\exp (\lambda /(\lambda-1))|$; hence, as in Section 5 ,

$$
a_{3}-\lambda a_{2}^{2}=1+2\left|\exp \frac{2 \lambda}{\lambda-1}\right|
$$

for the extremal function $f$. It should be expected that for all $\lambda$ of $F$ the corresponding extremal domain has a forked boundary.

There is a final remark. Let $V_{3}$ denote the coefficient body $\left\{\left(a_{2}, a_{3}\right): f \in S\right\}$, which is extensively discussed in Chapter XIII of [6]. Besides some limiting cases, the boundary points of $V_{3}$ consist of two big classes. The ones, say $\pi_{1}$, are yielded by "single arc functions", the other ones, say $\pi_{2}$, by "forked slit functions". In plates I and II of [6] the class $\pi_{1}$ is shown in yellow, the class $\pi_{2}$ in blue.

Clearly, functions maximizing $\left|a_{3}-\lambda a_{2}^{2}\right|$ over $S$, for some $\lambda \in C$, yield boundary points of $V_{3}$. For $\lambda$ real these points are made up by the real pairs $\left(a_{2}, a_{3}\right)$ and lie in $\pi_{2}$ (except for $\lambda=1$ ). It should be of some interest to see which part of the boundary of $V_{3}$ comes from the functions which maximize $\left|a_{3}-\lambda a_{2}^{2}\right|$ over $S$.

Added in proof. J. A. Jenkins [7] treated the same problem by his general coefficient theorem.

\section{References}

[1] Fekete, M., and G. Szegö: Eine Bemerkung über ungerade schlichte Funktionen. - J. London Math. Soc. 8, 1933, 85-89.

[2] Garabedian, P. M., and M. Schiffer: A coefficient inequality for schlicht functions. - Ann. of Math. 61, 1955, 116-136.

[3] Littlewnod, J. E., and R. E. A. C. Paley: A proof that an odd schlicht function has bounded coefficients. - J. London Math. Soc. 7, 1932, 167-169.

[4] Pólya, G., and G. Szegö: Problems and theorems in analysis, Vol. I, Problem 129. - SpringerVerlag, Berlin-Heidelberg-New York, 1972.

[5] Schaeffer, A. C., and D. C. Spencer: The coefficients of schlicht functions. - Duke Math. J. 10, 1943, 611-635.

[6] Schaeffer, A. C., and D. C. Spencer: Coefficient regions for schlicht functions. - American Mathematical Society Colloquium Publications, Vol. 35. American Mathematical Society, New York, N. Y., 1950.

[7] Jenkins, J. A.: On certain coefficients of univalent functions. - Analytic functions. Princeton University Press, Princeton, N. J., 1060, 159-194.

Eidgenössische Technische Hochschule

Mathematisches Institut

CH-8092 Zürich

Switzerland

Received 2 March 1984 